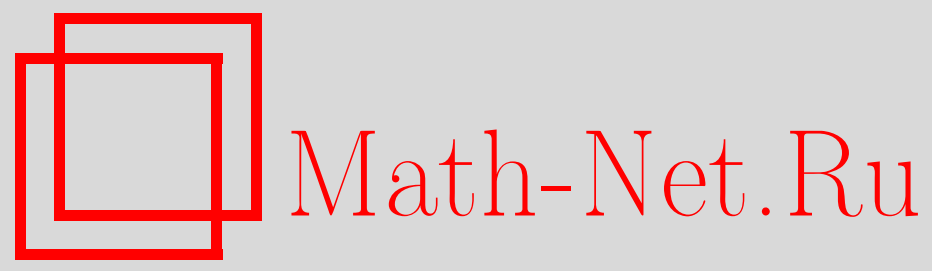

В. В. Стружанов, К. В. Бердников, Об определяющих соотношениях среды Генки для разупрочняющегося материала при диагональном тензоре деформаций, Вестн. Сам. гос. техн. ун-та. Сер. Физ.-мат. науки, 2012, выпуск 3(), 72-80

DOI: https://doi.org/10.14498/vsgtu1115

Использование Общероссийского математического портала Math-Net.Ru подразумевает, что вы прочитали и согласны с пользовательским соглашением

http://www.mathnet.ru/rus/agreement

Параметры загрузки:

IP: 54.89 .56 .158

26 апреля 2023 г., 16:45:14

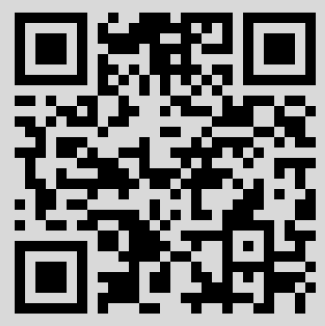


Вестн. Сам. гос. техн. ун-та. Сер. Физ.-мат. науки. 2012. № 3 (28). С. $72-80$

УДК 539.3

\section{ОБ ОПРЕДЕЛЯЮЩИХ СООТНОШЕНИЯХ СРЕДЫ ГЕНКИ ДЛЯ РАЗУПРОЧНЯЮЩЕГОСЯ МАТЕРИАЛА ПРИ ДИАГОНАЛЬНОМ ТЕНЗОРЕ ДЕФОРМАЦИЙ}

\section{В. В. Стружанов, К.В. Бердников}

Институт машиноведения УрО РАН,

620049, Россия, Екатеринбург, ул. Комсомольская, 34.

E-mails: stru@imach.uran.ru, kir.berdnikov@mail.ru

Рассматривается среда, деформаиии которой описываются диагональными компонентами тензора деформаций (в сферической системе координат). Полагается, что первый инвариант тензора деформаций не положителен. При этих ограничениях выписъваются определяющие соотношения Генки с учетом разупрочнения материала. Эти определяющие соотношения представляются как отображение пространства деформаций в пространство напряжений, матрииа Якоби которого в некоторых точках пространства деформаиий вырождена. Показано, что с помощъю данного отображения возможно найти реальное число деформированных состояний, отвечающих заданному тензору напряжений. Кроме того, выписаны уравнения инкременталъного закона пластичности, позволяющие по величине полных дебормаций найти их неупругие составляющие.

Ключевые слова: упрочнение, разупрочнение, среда Генки, пограничные состояния, матрицы Якоби и Гессе, неединственность равновесий, инкременталъный закон пластичности.

Введение. Включение в рассмотрение неустойчивых состояний материала [1-4] (стадии разупрочнения) приводит к тому, что определяющие соотношения уже не являются взаимно однозначным соответствием между деформациями и напряжениями. Такие определяющие соотношения относятся к классу дифференцируемых отображений с особенностями [5-7]. Их построение является достаточно сложной проблемой в механике деформируемого твердого тела. В данной работе модель среды Генки распространяется на стадию разупрочнения материала при следующих ограничениях: тензоры напряжений и деформаций имеют диагональный вид (в сферической системе координат), а объёмная деформация неположительна и, следовательно, связь между шаровыми тензорами напряжений и деформаций определяется законом Гука на всех стадиях деформирования. Полученные определяющие соотношения с особенностями позволяют найти реальное число деформированных состояний среды (как устойчивых, так и неустойчивых), отвечающих заданному тензору напряжений.

Приведён инкрементальный закон пластичности, дающий возможность вычислять неупругие составляющие деформаций. Рассмотрен пример для случая, когда две компоненты тензора деформаций равны, что наблюдается в задачах о расширении сферической полости в пространстве и деформировании сферического сосуда под действием внешнего давления.

Валерий Владимирович Стружанов (д.ф.-м.н., проф.), главный научный сотрудник, лаб. микромеханики материалов. Кирилл Вячеславович Бердников, инженер, лаб. микромеханики материалов. 
1. Свободная энергия. Пусть в сплошной среде реализовано напряжённодеформируемое состояние, которое в сферической системе координат определяется только диагональными компонентами тензоров напряжений и деформаций, а именно $\varepsilon_{r}, \varepsilon_{\theta}, \varepsilon_{\varphi}$ и $\sigma_{r}, \sigma_{\theta}, \sigma_{\varphi}$. Полагаем, что в изотермическом процессе активного деформирования приращение свободной энергии $d F$ отождествляется с элементарной работой напряжений (среда Генки [8]). В инвариантной форме записи находим, что $d F=3 \sigma_{0} d \varepsilon_{0}+T d \Gamma$ [8]. Здесь $\sigma_{0}=$ $=\left(\sigma_{r}+\sigma_{\theta}+\sigma_{\varphi}\right) / 3, \varepsilon_{0}=\left(\varepsilon_{r}+\varepsilon_{\theta}+\varepsilon_{\varphi}\right) / 3=\theta / 3(\theta$ - относительное изменение объёма материального элемента),

$$
T=\frac{1}{\sqrt{6}}\left[\left(\sigma_{r}-\sigma_{\varphi}\right)^{2}+\left(\sigma_{\varphi}-\sigma_{\theta}\right)^{2}+\left(\sigma_{\theta}-\sigma_{r}\right)^{2}\right]^{1 / 2}
$$

- интенсивность касательных напряжений,

$$
\Gamma=\sqrt{\frac{2}{3}}\left[\left(\varepsilon_{r}-\varepsilon_{\varphi}\right)^{2}+\left(\varepsilon_{\varphi}-\varepsilon_{\theta}\right)^{2}+\left(\varepsilon_{\theta}-\varepsilon_{r}\right)^{2}\right]^{1 / 2}
$$

- интенсивность деформаций сдвига [8].

Полагая пропорциональность шаровых тензоров и тензоров девиаторов напряжений и деформаций с коэффициентами пропорциональности $K^{s}$ и $G^{s}$, получаем

$$
d F=\frac{1}{3} K^{s} \theta d \theta+G^{s} \Gamma d \Gamma,
$$

т. е. $\sigma_{0}=K^{s} \varepsilon_{0}, T=G^{s} \Gamma$, а $K^{s}$ и $G^{s}$ - секущие модули. Если $\theta \leqslant 0$, то естественно считать, что на всех стадиях деформирования $\left(K^{s}=K=\mathrm{const}-\right.$ объёмный модуль в упругости). Таким образом, $G^{s}=G^{s}(\Gamma)-$ секущий модуль единой кривой $T \sim \Gamma$. В отличие от традиционной среды Генки, в данной работе полагаем, что единая кривая имеет ниспадающий участок, характеризующий стадию разупрочнения материала под действием сдвигающих деформаций.

Примерный вид такой кривой показан на рис. 1, где $\Gamma_{B}-$ интенсивность касательных деформаций, после достижения которой материал переходит на стадию разупрочнения, $\Gamma_{Z}$ - последняя точка единой кривой, в которой происходит разрушение материала, $G$ - касательный модуль в начале координат (модуль сдвига в упругости).

Процесс деформирования можно изобразить движением точки $(\theta, \Gamma)$ по некоторой кривой $L$ в двумерном

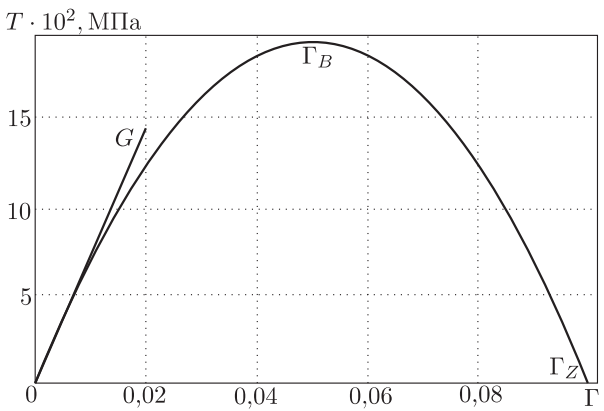

Рис. 1. Единая кривая с падающей ветвью евклидовом пространстве. Тогда свободная энергия

$$
F=\int_{L}\left(\frac{1}{3} K \theta d \theta+G^{s}(\Gamma) \Gamma\right) d \Gamma
$$


Очевидно, что подынтегральное выражение в данном случае является полным дифференциалом, и поэтому работа напряжений не зависит от вида пути деформирования. Восстанавливая функцию по её полному дифференциалу, имеем

$$
F(\theta, \Gamma)=F\left(\varepsilon_{r}, \varepsilon_{\theta}, \varepsilon_{\varphi}\right)=\frac{1}{6} K \theta^{2}+\int_{0}^{\Gamma} G^{s}(\Gamma) \Gamma d \Gamma
$$

2. Определяющие соотношения. Свободная энергия является потенциалом напряжений, т. е. $\sigma_{r}=\partial F / \partial \varepsilon_{r}, \sigma_{\varphi}=\partial F / \partial \varepsilon_{\varphi}, \sigma_{\theta}=\partial F / \partial \varepsilon_{\theta}$. Производя необходимые вычисления, находим соотношения среды Генки:

$$
\begin{gathered}
\sigma_{r}=\sigma_{r}\left(\varepsilon_{r}, \varepsilon_{\theta}, \varepsilon_{\varphi}\right)=\frac{1}{3} K \theta+2 G^{s}(\Gamma)\left(\varepsilon_{r}-\frac{1}{3} \theta\right), \\
\sigma_{\theta}=\sigma_{\theta}\left(\varepsilon_{r}, \varepsilon_{\theta}, \varepsilon_{\varphi}\right)=\frac{1}{3} K \theta+2 G^{s}(\Gamma)\left(\varepsilon_{\theta}-\frac{1}{3} \theta\right), \\
\sigma_{\varphi}=\sigma_{\varphi}\left(\varepsilon_{r}, \varepsilon_{\theta}, \varepsilon_{\varphi}\right)=\frac{1}{3} K \theta+2 G^{s}(\Gamma)\left(\varepsilon_{\varphi}-\frac{1}{3} \theta\right),
\end{gathered}
$$

причём $0 \leqslant G^{s}(\Gamma) \leqslant G$.

Уравнения (1) задают отображение из трехмерного евклидова пространства деформаций $\left(\varepsilon_{r}, \varepsilon_{\theta}, \varepsilon_{\varphi}\right)$ в трехмерное евклидово пространство напряжений $\left(\sigma_{r}, \sigma_{\theta}, \sigma_{\varphi}\right)$. Отображение является взаимно однозначным в том и только в том случае, когда матрица Якоби этого отображения невырождена (гомеоморфизм). Взаимная однозначность нарушается в окрестности тех точек пространства деформаций, где матрица Якоби вырождена.

Матрица Якоби отображения (1) имеет вид

$$
J=\left(\begin{array}{lll}
\partial \sigma_{r} / \partial \varepsilon_{r} & \partial \sigma_{r} / \partial \varepsilon_{\theta} & \partial \sigma_{r} / \partial \varepsilon_{\varphi} \\
\partial \sigma_{\theta} / \partial \varepsilon_{r} & \partial \sigma_{\theta} / \partial \varepsilon_{\theta} & \partial \sigma_{\theta} / \partial \varepsilon_{\varphi} \\
\partial \sigma_{\varphi} / \partial \varepsilon_{r} & \partial \sigma_{\varphi} / \partial \varepsilon_{\theta} & \partial \sigma_{\varphi} / \partial \varepsilon_{\varphi}
\end{array}\right)
$$

Отметим, что она совпадает с матрицей Гессе $H(F)$ функции $F$ :

$$
H(F)=\left(\begin{array}{lll}
C_{r r}^{p} & C_{r \theta}^{p} & C_{r \varphi}^{p} \\
C_{r \theta}^{p} & C_{\theta \theta}^{p} & C_{\theta \varphi}^{p} \\
C_{r \varphi}^{p} & C_{\theta \varphi}^{p} & C_{\varphi \varphi}^{p}
\end{array}\right)
$$

где

$$
\begin{gathered}
C_{r r}^{p}=\frac{\partial^{2} F}{\partial \varepsilon_{r}^{2}}=\frac{\partial \sigma_{r}}{\partial \varepsilon_{r}}=A(\Gamma)+B(\Gamma) M_{r}^{2}(\varepsilon), \\
C_{\theta \theta}^{p}=\frac{\partial^{2} F}{\partial \varepsilon_{\theta}^{2}}=\frac{\partial \sigma_{\theta}}{\partial \varepsilon_{\theta}}=A(\Gamma)+B(\Gamma) M_{\theta}^{2}(\varepsilon), \\
C_{\varphi \varphi}^{p}=\frac{\partial^{2} F}{\partial \varepsilon_{\varphi}^{2}}=\frac{\partial \sigma_{\varphi}}{\partial \varepsilon_{\varphi}}=A(\Gamma)+B(\Gamma) M_{\varphi}^{2}(\varepsilon), \\
C_{r \theta}^{p}=\frac{\partial^{2} F}{\partial \varepsilon_{r} \partial \varepsilon_{\theta}}=\frac{\partial \sigma_{r}}{\partial \varepsilon_{\theta}}=D(\Gamma)+B(\Gamma) M_{r}(\varepsilon) M_{\theta}(\varepsilon),
\end{gathered}
$$




$$
\begin{gathered}
C_{r \varphi}^{p}=\frac{\partial^{2} F}{\partial \varepsilon_{r} \partial \varepsilon_{\varphi}}=\frac{\partial \sigma_{r}}{\partial \varepsilon_{\varphi}}=D(\Gamma)+B(\Gamma) M_{r}(\varepsilon) M_{\varphi}(\varepsilon), \\
C_{\theta \varphi}^{p}=\frac{\partial^{2} F}{\partial \varepsilon_{\theta} \partial \varepsilon_{\varphi}}=\frac{\partial \sigma_{\theta}}{\partial \varepsilon_{\varphi}}=D(\Gamma)+B(\Gamma) M_{\theta}(\varepsilon) M_{\varphi}(\varphi) ; \\
A(\Gamma)=\frac{1}{3}\left(K+4 G^{s}\right), \quad B(\Gamma)=\frac{4}{9 \Gamma} \frac{d G^{s}(\Gamma)}{d \Gamma}, \quad D(\Gamma)=\frac{1}{3}\left(K-2 G^{s}\right), \\
M_{r}(\varepsilon)=2 \varepsilon_{r}-\varepsilon_{\theta}-\varepsilon_{\varphi}, \quad M_{\theta}(\varepsilon)=2 \varepsilon_{\theta}-\varepsilon_{r}-\varepsilon_{\varphi}, \quad M_{\varphi}(\varepsilon)=2 \varepsilon_{\varphi}-\varepsilon_{r}-\varepsilon_{\theta} .
\end{gathered}
$$

3. Критические точки и критические значения определяющих соотношений. Найдём точки в пространстве деформаций, где матрица Якоби (2) отображения (1) вырождена. Вычислим определитель матрицы Якоби:

$$
\begin{aligned}
\operatorname{det}(J)=A^{3}+2 & D^{3}+A^{2} B\left(M_{\varphi}^{2}+M_{\theta}^{2}+M_{r}^{2}\right)- \\
& \quad 2 A D B\left(M_{\varphi} M_{\theta}+M_{r} M_{\theta}+M_{\varphi} M_{r}\right)+ \\
+ & D^{2} B\left(-M_{\varphi}^{2}-M_{\theta}^{2}-M_{r}^{2}+2 M \varphi M \theta+2 M_{r} M_{\theta}+2 M_{\varphi} M_{r}\right) .
\end{aligned}
$$

Далее подставляя функции $A(\Gamma), B(\Gamma), D(\Gamma), M_{r}(\varepsilon), M_{\theta}(\varepsilon), M_{\varphi}(\varepsilon)$ в явном виде и учитывая выражений для $\Gamma$, получаем

$$
\operatorname{det}(J)=4 K G^{p}(\Gamma) G^{s}(\Gamma) .
$$

Таким образом, матрица Якоби вырождается тогда, когда касательный модуль единой кривой $G^{p}$ обращается в нуль (наивысшая точка кривой, где $\Gamma=\Gamma_{B}$ ), или при $G^{s}=0$ (точка кривой, где $\Gamma=\Gamma_{Z}$ ). Равенства $\Gamma=\Gamma_{B}$ и $\Gamma=\Gamma_{Z}$ определяют два цилиндра (цилиндры Мизеса при $\theta \leqslant 0$ ) в пространстве главных деформаций, образующие которых ортогональны девиаторной плоскости $\left(\varepsilon_{r}+\varepsilon_{\theta}+\varepsilon_{\varphi}=0\right)$, проходящей через начало координат и равнонаклонённой к координатным осям. Точки в пространстве деформаций, расположенные на поверхностях этих цилиндров, есть критические

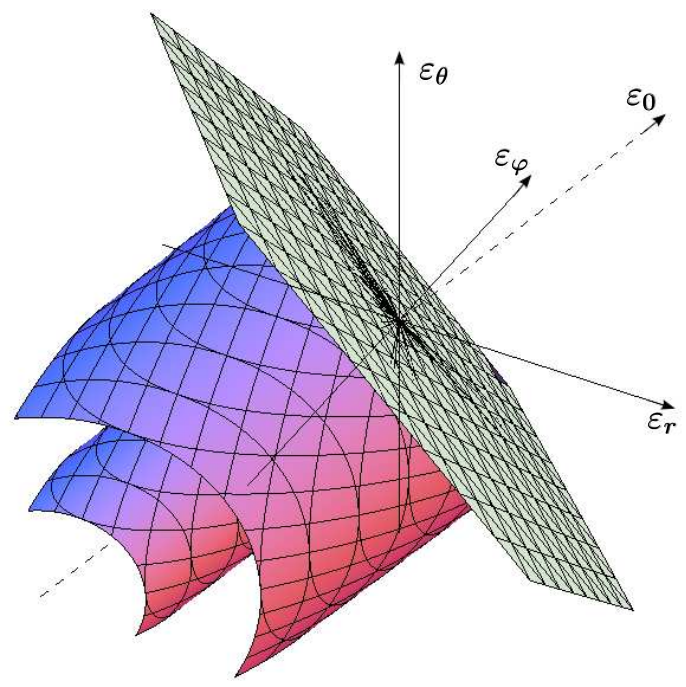

Рис. 2. Поверхности критических точек отображения (1) в пространстве деформаций 
точки отображения (1) [5]. На рис. 2 указанные выше цилиндры построены для единой кривой (см. рис. 1$)$, заданной уравнением $T(\Gamma)=\mu(\Gamma) \Gamma$, где $\mu(\Gamma)=G(1-10 \Gamma), G=76 \cdot 10^{3}$ МПа. Точкам, расположенным внутри внутреннего цилиндра, отвечают устойчивые состояния материала (упрочнение), точки поверхности внутреннего цилиндра - точки пограничных состояний, разделяющие упрочнение от разупрочнения. Точкам, расположенным между цилиндрами, отвечает разупрочнение материала. Точки поверхности внешнего цилиндра - точки разрушения, которое происходит под действием деформаций сдвига и превращает материал в аналог сыпучей среды, не способной сопротивляться сдвигам.

Посредством соотношений Генки (1), где полагаем $K=5 \cdot 10^{5}$ МПа, отобразим полученные цилиндры (см. рис. 2) в пространство напряжений. Внешний цилиндр, соответствующий пограничному состоянию материала между разупрочнением и разрушением, в пространстве напряжений переходит в нормаль к девиаторной плоскости $\left(\sigma_{r}+\sigma_{\theta}+\sigma_{\varphi}=0\right)$, проходящую через начало координат. Цилиндр, разделяющий состояние упрочнения материала от состояния разупрочнения, отображается в цилиндр, образующие которого, как и в пространстве деформаций, ортогональны девиаторной плоскости, изображён на рис. 3. Точки поверхности цилиндра I и прямой II - критические значения отображения (1) [5].

Итак, если вести нагружение посредством задания значений для деформаций, то сначала путь деформирования будет располагаться внутри первого (внутреннего) цилиндра (см. рис. 2) и материал находится на стадии упрочнения. Компоненты тензора напряжений определяют точки в пространстве напряжений, расположенные внутри цилиндра I (см. рис. 3). После пересечения путём деформирования поверхности внутреннего цилиндра (см. рис. 2) материал переходит на стадию разупрочнения. При этом путь в пространстве напряжений, достигнув поверхности цилиндра I (см. рис. 3), поворачивает обратно. Отображение (1) снова определяет точки, расположенные внутри цилиндра I. Когда путь деформирования пересечёт поверхность внешнего ци-

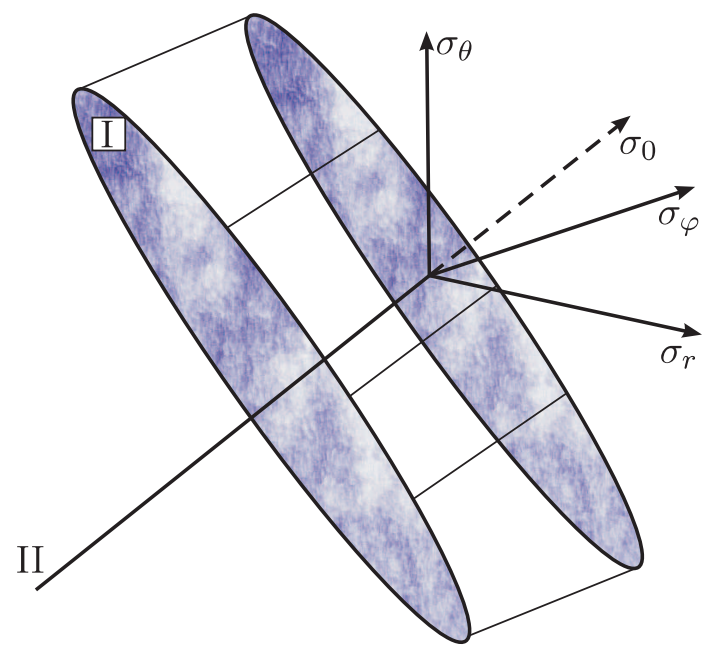

Рис. 3. Критические значения отображения (1): точки поверхности цилиндра (I) и прямой (II) 
линдра (разрушение от сдвигов), тогда путь нагружения остановится в какойлибо точке прямой II (см. рис. 3).

Таким образом, каждой точке в пространстве напряжений, расположенной внутри цилиндра I, отображение (1) определяет два деформированных состояния: одно - состояние упрочнения, другое - состояние разупрочнения.

4. Частный случай. Рассмотрим частный случай, предполагая выполнение равенства $\varepsilon_{\theta}=\varepsilon_{\varphi}$, которое справедливо в задачах о расширении сферической полости в пространстве и деформировании толстостенного сферического сосуда под действием равномерного внешнего давления. Теперь удобно рассматривать двумерное пространство деформаций $\left(\varepsilon_{r}, \varepsilon_{\theta}\right)$ и напряжений $\left(\sigma_{r}, \sigma_{\theta}\right)$. В этом случае области вырожденности представляют собой прямые, образованные пересечением цилиндров на рис. 2 и плоскости $\varepsilon_{\theta}=\varepsilon_{\varphi}$. На рис. 4 аналогично общему случаю, рассмотренному ранее, I - область упрочнения, II - область разупрочнения. Понятно, что при отображении областей вырожденности в пространство напряжений соотношениями (1) получаются прямые, которые являются пересечениями цилиндра и нормали к девиаторной плоскости $\left(\sigma_{r}+\sigma_{\theta}+\sigma_{\varphi}=0\right)$, построенной в начале координат (см. рис. 3$)$ с плоскостью $\sigma_{\theta}=\sigma_{\varphi}$.

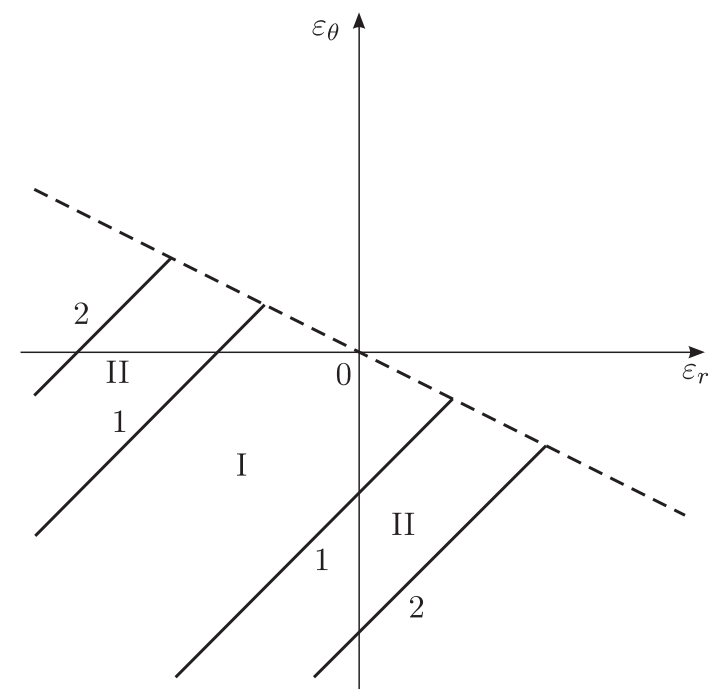

Рис. 4. Критические точки отображения (1) при $\varepsilon_{\theta}=\varepsilon_{\varphi}$ (прямые 1 и 2 )

5. Определение приращений пластических деформаций. Наконец определим пластические составляющие приращения полной деформации, используя то обстоятельство, что разгрузка происходит по линейному закону с модулями $K$ и $G$.

Пусть в некотором деформированном состоянии материал имеет инкрементальные модули, заданные компонентами матрицы $C^{p}$, совпадающей с матрицей Гессе функции свободной энергии $H(F)$. Возмутим это состояние посредством задания приращения полных деформаций $\overrightarrow{d \varepsilon}$. Приращение пластических деформаций определяем с использованием инкрементального закона пластичности [9]

$$
\overrightarrow{d \varepsilon^{p}}=\left(I-S C^{p}\right) \overrightarrow{d \varepsilon}
$$


где $I$ - единичная матрица третьего порядка; $\overrightarrow{d \varepsilon^{p}}-$ вектор-столбец с компонентами $d \varepsilon_{r}^{p}, d \varepsilon_{\theta}^{p}, d \varepsilon_{\varphi}^{p} ; \overrightarrow{d \varepsilon}$ - вектор-столбец приращений полных деформаций; $S$ - матрица коэффициентов упругой податливости:

$$
S=\frac{1}{E}\left(\begin{array}{ccc}
1 & -\nu & -\nu \\
-\nu & 1 & -\nu \\
-\nu & -\nu & 1
\end{array}\right)
$$

$E$ - модуль Юнга; $\nu$ - коэффициент Пуассона. Отсюда

$$
\begin{aligned}
d \varepsilon_{r}^{p}= & {\left[1-E^{-1}\left(C_{r r}^{p}-\nu C_{r \theta}^{p}-\nu C_{r \varphi}^{p}\right)\right] d \varepsilon_{r}-} \\
& -E^{-1}\left(C_{r \theta}^{p}-\nu C_{\theta \theta}^{p}-\nu C_{\theta \varphi}^{p}\right) d \varepsilon_{\theta}-E^{-1}\left(C_{r \varphi}^{p}-\nu C_{\theta \varphi}^{p}-\nu C_{\varphi \varphi}^{p}\right) d \varepsilon_{\varphi}, \\
d \varepsilon_{\theta}^{p}= & {\left[1-E^{-1}\left(C_{\theta \theta}^{p}-\nu C_{r \theta}^{p}-\nu C_{\theta \varphi}^{p}\right)\right] d \varepsilon_{\theta}-} \\
& \quad-E^{-1}\left(C_{r \theta}^{p}-\nu C_{r r}^{p}-\nu C_{r \varphi}^{p}\right) d \varepsilon_{r}-E^{-1}\left(C_{\theta \varphi}^{p}-\nu C_{r \varphi}^{p}-\nu C_{\varphi \varphi}^{p}\right) d \varepsilon_{\varphi}, \\
d \varepsilon_{\varphi}^{p}= & {\left[1-E^{-1}\left(C_{\varphi \varphi}^{p}-\nu C_{r \varphi}^{p}-\nu C_{\theta \varphi}^{p}\right)\right] d \varepsilon_{\varphi}-} \\
& \quad-E^{-1}\left(C_{r \varphi}^{p}-\nu C_{r \varphi}^{p}-\nu C_{r \theta}^{p}\right) d \varepsilon_{r}-E^{-1}\left(C_{\theta \varphi}^{p}-\nu C_{r \theta}^{p}-\nu C_{\theta \theta}^{p}\right) d \varepsilon_{\theta} .
\end{aligned}
$$

После подстановки всех значений можно убедиться в том, что $d \varepsilon_{r}^{p}+d \varepsilon_{\theta}^{p}+$ $+d \varepsilon_{\varphi}^{p}=0$, т. е. при неизменном модуле $K$ объёмная деформация сохраняет упругость на всех стадиях деформирования. Тогда

Наиболее простой вид данные выражения принимают при условии $\varepsilon_{\theta}=\varepsilon_{\varphi}$.

$$
d \varepsilon_{r}^{p}=-\frac{2}{3}\left(1-\frac{G^{p}}{G}\right) d \gamma, \quad d \varepsilon_{\theta}^{p}=d \varepsilon_{\varphi}^{p}=\frac{1}{3}\left(1-\frac{G^{p}}{G}\right) d \gamma
$$

где $d \gamma=d \varepsilon_{\varphi}-d \varepsilon_{r}-$ приращение максимального сдвига. Очевидно, что

$d \varepsilon_{r}^{p}+d \varepsilon_{\theta}^{p}+d \varepsilon_{\varphi}^{p}=0, \quad d \gamma^{p}=\left(1-\frac{G^{p}}{G}\right) d \gamma, \quad d \varepsilon_{r}^{p}=-\frac{2}{3} d \gamma^{p}, \quad d \varepsilon_{\theta}^{p}=d \varepsilon_{\varphi}^{p}=\frac{1}{3} d \gamma^{p}$

Из полученных формул следует, что при $G^{p}>0$ только часть подведенной энергии расходуется на образование пластических (неупругих) деформаций. Когда $G^{p}<0$, на образование пластических деформаций уходит вся подведенная энергия и еще часть упругой энергии, накопленной в материале.

Заключение. При некоторых предположениях для среды Генки, единая кривая которой имеет падающую ветвь, получены определяющие соотношения. Исследованы их свойства как отображения пространства деформаций в пространство напряжений. С использованием инкрементального закона пластичности получены выражения для приращения пластических (неупругих) деформаций.

Работа выполнена по совместному проекту УрО РАН и СО РАН (проект № 12-C-1-1024). 


\section{БИБЛИОГРАФИЧЕСКИЙ СПИСОК}

1. Радченко В. П., Небогина E. В., Андреева E. А. Структурная модель разупрочняющегося при ползучести материала в условиях сложного напряженного состояния // Becmн. Сам. гос. техн. ун-та. Сер. Физ.-мат. науки, 2009. №1(18). С. 75-84. [Radchenko V. P., Nebogina E. V., Andreeva E. A. Structural Model of Material Softening at Creep under Complex Stress Conditions // Vestn. Samar. Gos. Tekhn. Univ. Ser. Fiz.-Mat. Nauki, 2009. no. 1(18). Pp. 75-84].

2. Кадашевич Е.Ю., Помыткин С. П. Исследование одноосного и двуосного нагружения разупрочняющихся материалов по эндохронной теории неупругости // Becmн. Cам. гос. техн. ун-та. Сер. Физ.-мат. науки, 2012. №1(26). С. 110-115. [Kadashevich Yu. I., Pomytkin S.P. Investigation of uniaxial and biaxial loadings of softening materials in endochronic theory of inelasticity // Vestn. Samar. Gos. Tekhn. Univ. Ser. Fiz.-Mat. Nauki, 2012. no. 1(26). Pp. 110-115].

3. Стружанов В. В., Башуров Вяч. В. Модификационная модель Мазинга // Вестн. Сам. гос. техн. ун-та. Сер. Физ.-мат. науки, 2007. №1(14). С. 29-39. [Struzhanov V. V., Bashurov Vyach. V. The modified Masing model// Vestn. Samar. Gos. Tekhn. Univ. Ser. Fiz.-Mat. Nauki, 2007. Vol. 1(14). Pp. 29-39].

4. Стружанов В. В. Упругопластическая среда с разупрочнением. Сообщение 1. Свойства материала и инкрементальный закон пластичности при растяжении // Becmн. Сам. гос. техн. ун-та. Сер. Физ.-мат. науки, 2006. № 42. С. 49-61. [V. V. Struzhanov Elastoplastic medium with a softening. Part 1. Material properties and incremental plasticity law is in tension // Vestn. Samar. Gos. Tekhn. Univ. Ser. Fiz.-Mat. Nauki, 2006. no.42. Pp. 49-61].

5. Арнольд В.И., Варченко А.Н., Гусейн-Заде С.М. Особенности дифференцируемых отображений. Т. 1: Классификация критических точек, каустик и волновых фронтов. M.: Наука, 1982. 304 с.; англ. пер.: Arnold V.I., Varchenko A.N., Gusein-Zade S.M. Singularities of Differentiable Maps. Vol. 1: The Classification of Critical Points Caustics, Wave Fronts. Boston: Birkhäuser, 1985. 396 pp. (Monographs in Mathematics. Vol. 82)

6. Стружанов В.В., Просвиряков Е.Ю. Растяжение с кручением. Сообщение 2: Устойчивость процесса деформирования образца в механической системе. Жёсткое и мягкое нагружения // Вестн. Сам. гос. техн. ун-та. Сер. Физ.-мат. науки, 2008. № 2(17). C. 77-86. [Struzhanov V. V., Prosviryakov E. Yu. Tension with torsion. Part 2. Deformation process stability of a sample in a mechanical system. Rigid and soft loadings // Vestn. Samar. Gos. Tekhn. Univ. Ser. Fiz.-Mat. Nauki, 2008. no. 2(17). Pp. 77-86].

7. Стружанов B. В., Бахарева E.A. Математические методы в теории чистого изгиба прямоугольных балок из разупрочняющегося материала с симметричной диаграммой растяжения-сжатия// Вычисл. мех. сплош. сред, 2012. Т. 5, №2. С. 158-167. [Struzhanov V.V., Bakhareva E. A. Mathematical methods in the theory of pure bending of rectangular beams made of weakening material with symmetric stress-strain diagram // Computational Continuum Mechanics, 2012. Vol. 5, no. 2. Pp. 158-167].

8. Луръе А.И. Теория упругости. М.: Наука, 1970. 939 с. [Lurie A. I. Theory of Elasticity. Moscow: Nauka, 1970. 939 pp.]

9. Стружанов В. В., Миронов В. И. Деформационное разупрочнение материала в элементах конструкций. Екатеринбург: УрО РАН, 1995. 192 с. [Struzhanov V. V., Mironov V.I. Strain softening of the material in structural elements. Yekaterinburg: UrO RAN, 1995. 191 pp.]

Поступила в редакцию 20/VII/2009;

в окончательном варианте - 11/VIII/2012. 
MSC: $74 \mathrm{C} 10$

ON DEFINING RELATIONS FOR THE HENCKY ENVIRONMENT OF SOFTENING OF THE MATERIAL UNDER DIAGONAL STRESS TENSOR

\section{V. Struzhanov, K. V. Berdnikov}

Institute of Engineering Science, Ural Branch of RAS, 34, Komsomolskaya st., Ekaterinburg, 620049, Russia.

E-mails: stru@imach.uran.ru, kir.berdnikov@mail.ru

Medium which strains are described by diagonal components of the strain tensor is considered (in spherical coordinate system). It is assumed that the first invariant of the strain tensor is not positive. Under these restrictions Hencky defining relations with regard to softening of material are written. These defining relations are represented as map of strain space in the stress space. Jacobi matrix of this map is singular in some points in strain space. It is shown that using this map it is possible to find the objective number of deformed states corresponding to a given strain tensor. Also the equations of incremental plasticity law are written. These equations allow us to find the inelastic strain by the total strain.

Key words: hardening, softening, Hencky enviroment, boundary states, Jacobi matrix and Hesse matrix, non-uniqueness of equilibria, incremental plasticity law.

Original article submitted 20/VII/2009; revision submitted 11/VIII/2012.

Valery V. Struzhanov (Dr. Sci. (Phys. \& Math.)), Chief researcher, Lab. of Matherial Micromechanics. Kirill V. Berdnikov, Engineer, Lab. of Matherial Micromechanics. 